\title{
Thyroid Carcinoma Pattern Presentation According to Age
}

\author{
Fábio Muradás Girardi ${ }^{1}$ \\ ${ }^{1}$ Department of Head and Neck Surgery, Hospital Santa Rita, \\ Complexo Hospitalar Santa Casa de Porto Alegre, Porto Alegre, Brazil \\ Int Arch Otorhinolaryngol 2017;21:38-41. \\ Address for correspondence Fábio Muradás Girardi, MD, MSc, \\ Department of Head and Neck Surgery, Hospital Santa Rita, Complexo \\ Hospital Santa Casa de Porto Alegre, Independência, 354, 901 Porto \\ Alegre, RS 90035070, Brazil (e-mail: fabiomgirardi@gmail.com).
}

\begin{abstract}
Introduction Patients with thyroid cancer in different age groups present with different prognosis.

Objective The objective of this study is to analyze the clinicopathological pattern of thyroid carcinoma presentation according to age groups.

Methods This is a retrospective study. From 2000 to 2010, 623 patients underwent thyroidectomy for cancer in our institution, with 596 enrolled. Patients were divided into groups of 10 years and then in four age subgroups ( $\leq 24,25-44,45-64$, and $\geq 65$ years) for statistical analysis. We compared age, gender, and histopathological characteristics between groups.

Results Individuals belonging to the earlier age group presented with a highest prevalence of neuro-vascular invasion, capsular invasion and lymph node metastasis. Together with individuals of advanced age, that group also had larger tumor diameter and higher prevalence of extra-glandular disease. Even when analyzed only cases with well-differentiated carcinoma, younger individuals remain with a highest prevalence of lymph node metastasis, neuro-vascular invasion and larger tumor diameter.

\section{Keywords}

- papillary carcinoma

- thyroid neoplasms

- age

Conclusion We observed a distinct pattern of clinicopathological manifestation of thyroid cancer according to age. Individuals belonging to age extremes resemble in several pathological features, and young people usually present with more aggressive disease characteristics.
\end{abstract}

\section{Introduction}

Thyroid cancer is the only one that takes into account the variable age in the staging of the disease. ${ }^{1}$ Studies have shown that the age of the patient at the clinical presentation is a variable independently associated with prognosis., 2,3 Although for reasons not completely understood, younger individuals usually have better survival rates. Apparently, something intrinsic to cancer or the treatment is dependent on the age of the patient. Some studies have shown that the clinicopathological presentation does not necessarily follow the prognosis of patients. Younger individuals usually present with clinical and pathological factors traditionally associated with worse outcomes, despite belonging to the group with

received

January 19, 2016

accepted

June 3, 2016

published online

July 12, 2016 better survival. ${ }^{4-6}$ Our goal was to analyze the pattern of presentation of thyroid cancer according to age at onset of the disease through a large institutional series.

\section{Methods}

\section{Patients}

We reviewed histopathological records of all patients who underwent thyroidectomy with final histological diagnosis of thyroid cancer from June 2000 to December 2010 at our institution. All patients underwent clinical assessment and ultrasound preoperatively. Relevant cases underwent cytological evaluation of thyroid nodules by Fine Needle Aspiration (FNA). Neck dissection of central or lateral
Copyright $(2017$ by Thieme-Revinter

Publicações Ltda, Rio de Janeiro, Brazil
License terms

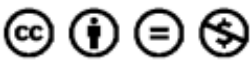


compartment in thyroid cancer cases is not performed electively in our institution, being reserved for cases clinically or ultrasonographically suspected for lymph node metastasis.

Out of 623 thyroid cancer confirmed cases, we included only cases with complete clinical e pathological information, treated with curative intent. We excluded 4 cases without information of age, 17 patients without tumor diameter information, and 6 cases with unresectable disease ( 4 cases of anaplastic carcinoma in patients between 64 and 83 years old; one case of medullary carcinoma in a patient of 68 years old; and 1 case of follicular carcinoma in a patient 80 years old). In all, 596 cases met the inclusion criteria. The cases included were distributed according to age groups of 10 years. After preliminary analysis, the sample was subdivided into four subgroups. Gender, age, and pathological data were compared among them. We performed a secondary analysis only with cases of well-differentiated thyroid cancer (561 cases of papillary and follicular carcinomas). The following parameters were entered into a specific database (Microsoft Excel®, 2013 version, Microsoft Corporation, Redmond, WA, USA): age, gender, lymph node dissection data, and detailed description of histopathological staging.

\section{Definitions and Pathology}

Tumors were considered as multifocal when more than one tumor focus was present in the gland. The clinical and pathological staging was performed according to the seventh edition of the American Joint Committee on Cancer staging system pTNM. Lymph node status was defined by pathological evidence of metastasis in lymph nodes removed. Extraglandular involvement was defined based on evidence of tumor infiltration beyond the glandular capsule on microscopic examination. The same researcher collected all data (Girardi FM) and the same pathologist (Barra MB) performed all pathological reviews.

\section{Statistics and Ethical Aspects}

We used descriptive analysis to summarize data. We performed the Kolmogorov-Smirnov test to evaluate normality of variable diameter. Age information was expressed as mean and standard deviation. Diameter information was expressed in median, minimum, and maximum value. Categorical variables were expressed as absolute and relative frequencies. We used Kruskal-Wallis test comparing diameter data, followed by post hoc analysis, and the nonparametric chi-square test on the comparison of categorical variables. We performed statistical analysis using SPSS software version 15.0 (SPSS Inc., Chicago, IL). All tests considered a significance level of 5\%. The authors ensure the preservation of the data and the confidentiality of the material. As there were no interventions, we did not apply the informed consent. The Research Ethics Committee of our institution (Project No. 3483/11) approved the project.

\section{Results}

Of the 623 cases of thyroid cancer treated with thyroidectomy between the years 2000 to 2010,596 patients (95.6\%) met the inclusion criteria. Of the total, 475 cases (79.6\%) were women. The male-to-female ratio was $1: 3.9$. The mean age was 48.14 (14.71) years, ranging 12-90 years. Peak occurrence was between 51 and 60 years. The distribution of cases by age group was $12,71,103,144,147,78$, and 41 , among patients $<20,21-30,31-40,41-50,51-60,61-70$, and $\geq 71$ years old, respectively. Papillary carcinoma was prevalent in all age groups, although it showed a higher relative frequency between 31 and 50 years (see - Fig. 1). Among the elders, papillary carcinoma has partly lost space to follicular, poorly differentiated, and anaplastic carcinoma. Capsular invasion, extra-glandular disease, and neuro-vascular invasion were more frequent at age extremes. On the other hand, multifocality was less frequent in these age groups. The zenith and the nadir for frequency of cervical lymph node metastasis was between 21 and 30 years and between 61 and 70 years old, respectively.

When analyzing the four age groups ( $\leq 24$ years; $25-44$ years; $45-64$ years, and $\geq 65$ years), we found no statistical significance in the comparison of genders and multifocality (see - Table 1). However, the four groups showed differences regarding tumor size, histology, presence of neuro-vascular invasion, capsular invasion, extra-glandular disease, cervical lymph node metastasis, and disease in the lateral neck compartment. Younger individuals' tumors presented with characteristics that are more aggressive: a greater association with neuro-vascular invasion, capsular invasion, and lymph node metastasis. Along with older individuals, young people's tumors showed greater tumor diameter and higher prevalence of extra-glandular disease. Even when analyzing only cases of well-differentiated thyroid carcinoma, lymph node metastasis, greater tumor diameter, and higher association with neuro-vascular invasion remain more frequent among young people's tumors (see - Table 2 ).

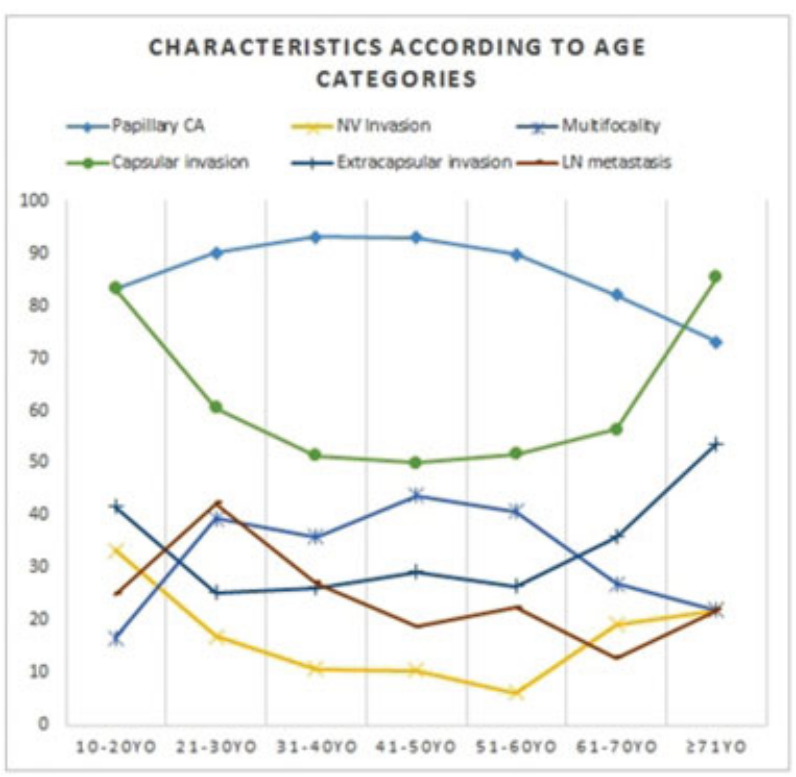

Fig. 1 Characteristics of patients with thyroid carcinoma (relative frequencies) according to age categories. Abbreviations: YO, years old. 
Table 1 Pattern of histopathological presentation of thyroid carcinoma according to age

\begin{tabular}{|c|c|c|c|c|c|c|c|c|c|c|c|c|}
\hline & & \multicolumn{10}{|c|}{ Age } & \multirow[t]{2}{*}{$p$ value } \\
\hline & & \multicolumn{2}{|c|}{$\leq 24$ yo } & \multicolumn{2}{|c|}{$25-44$ yo } & \multicolumn{2}{|c|}{$45-64$ yo } & \multicolumn{2}{|c|}{$\geq 65$ yo } & \multicolumn{2}{|c|}{ Total } & \\
\hline \multicolumn{2}{|l|}{ Mean (SD) } & \multicolumn{2}{|c|}{$20.15(3.13)$} & \multicolumn{2}{|c|}{$35.85(5.77)$} & \multicolumn{2}{|c|}{$53.27(5.16)$} & \multicolumn{2}{|c|}{71.33 (5.79) } & \multicolumn{2}{|c|}{$48.14(14.71)$} & - \\
\hline \multicolumn{2}{|c|}{$\begin{array}{l}\text { Tumoral Diameter } \\
\text { Median (Min-Max) }\end{array}$} & \multicolumn{2}{|c|}{$2(0.1-7)$} & \multicolumn{2}{|c|}{$1.5(0.1-7)$} & \multicolumn{2}{|c|}{$1.1(0.04-15)$} & \multicolumn{2}{|c|}{$2.1(0.1-12)$} & \multicolumn{2}{|c|}{$1.3(0.04-15)$} & $<0.001$ \\
\hline & & $\mathrm{N}$ & $\%$ & $N$ & $\%$ & $\mathrm{~N}$ & $\%$ & $\mathrm{~N}$ & $\%$ & $\mathrm{~N}$ & $\%$ & - \\
\hline \multirow[t]{2}{*}{ Gender } & Male & 5 & 15.1 & 40 & 19.5 & 51 & 18.9 & 25 & 28.0 & 121 & 20.3 & \multirow[t]{2}{*}{0.235} \\
\hline & Female & 28 & 84.8 & 165 & 80.4 & 218 & 81.0 & 64 & 71.9 & 475 & 79.6 & \\
\hline \multirow[t]{5}{*}{ Histology (CA) } & Papillary & 26 & 78.7 & 192 & 93.6 & 248 & 92.1 & 64 & 71.9 & 530 & 88.9 & \multirow[t]{5}{*}{$<0.001$} \\
\hline & Follicular & 3 & 9.0 & 6 & 2.9 & 11 & 4.0 & 11 & 12.3 & 31 & 5.2 & \\
\hline & Medullary & 1 & 3.0 & 7 & 3.4 & 6 & 2.2 & 5 & 5.6 & 19 & 3.1 & \\
\hline & PD & 3 & 9.0 & 0 & 0.0 & 1 & 0.3 & 2 & 2.2 & 6 & 1.0 & \\
\hline & Anaplastic & 0 & 0.0 & 0 & 0.0 & 3 & 1.1 & 7 & 7.8 & 10 & 1.6 & \\
\hline \multicolumn{2}{|c|}{$\begin{array}{l}\text { Neural or lymphovascular } \\
\text { invasion }\end{array}$} & 9 & 27.2 & 24 & 11.7 & 24 & 8.9 & 18 & 20.2 & 75 & 12.5 & 0.002 \\
\hline \multicolumn{2}{|l|}{ Multifocality } & 10 & 30.3 & 75 & 36.5 & 100 & 37.1 & 24 & 26.9 & 209 & 35.0 & 0.288 \\
\hline \multicolumn{2}{|c|}{ Gland capsule invasion } & 26 & 78.7 & 107 & 52.1 & 140 & 52.0 & 50 & 56.1 & 323 & 54.1 & 0.029 \\
\hline \multicolumn{2}{|c|}{ Extraglandular invasion } & 14 & 42.4 & 52 & 25.3 & 77 & 28.6 & 38 & 42.6 & 181 & 30.3 & 0.009 \\
\hline \multicolumn{2}{|l|}{ Positive neck LN } & 13 & 39.3 & 56 & 27.3 & 58 & 21.5 & 13 & 14.6 & 140 & 23.4 & 0.013 \\
\hline \multicolumn{2}{|c|}{$\begin{array}{l}\text { Lateral compartment positive } \\
\text { LN }\end{array}$} & 4 & 12.1 & 29 & 14.1 & 17 & 6.3 & 9 & 10.1 & 59 & 9.8 & 0.042 \\
\hline
\end{tabular}

Abbreviations: CA, Carcinoma; LN, Lymph node; PD, Poorly differentiated; yo, years old.

Note: Variables were expressed in absolute $(\mathrm{N})$ and relative frequencies (\%); Mean (Standard Deviation) or Median (Minimum to maximum) were used according to appropriate; diameter was expressed in centimeters; $p=$ level of significance. Post-hoc analysis followed Kruskal-Wallis test when analyzing diameter. Statistically significant differences were observed between: $25-44$ yo and $45-64$ yo $(p=0.014)$; $45-64$ yo and $\geq 65$ yo $(p<0.001)$; $\leq 24$ yo and $45-64$ yo $(p=0.005)$.

\section{Discussion}

Although the prognosis of well-differentiated thyroid carcinomas with localized disease is excellent, in situations with regional disease or distant metastasis, survival is dependent on age. ${ }^{7}$ In two studies by Ito et al, both among cases of papillary and follicular thyroid carcinoma, there was higher disease-specific survival among younger patients, although this group was more susceptible to regional and distant recurrences. ${ }^{5,6}$ With some differences in age divisions among studies, younger patients presented with larger diameter tumors and a higher prevalence of lymph node metastasis in the central compartment. Unlike our study, the prevalence of extra-glandular disease was higher in patients with advanced age. In our study, the lowest relative frequency of papillary carcinoma in the age extremes was due to a higher frequency of poorly differentiated carcinoma among young people and undifferentiated carcinoma in the elderly, as well as higher rate of follicular carcinomas in both subgroups. Differences in histology among different childhood thyroid cancer populations were already described, suggesting a different process of carcinogenesis, involving such environmental and hereditary factors. ${ }^{8}$ Although the histological composition may partly explain differences in other pathological characteristics of each age group, similar differences were also present when analyzing only cases of well-differ- entiated carcinoma, which minimize the possibility of sampling bias.

It remains unclear why younger individuals, despite the aggressive presentation of the disease, often have better survival rates. Perhaps the follow-up period needs to be more than 20 years to observe any impact on disease-specific mortality. However, surgery with complete resection, complemented by hormonal suppression and radioiodine therapy, usually ensures prolonged survival, even with advanced disease. Based on the analysis of healthy individuals, with increasing age the usual decline of immune response and post-menopausal hormonal changes could also collaborate with the mortality differences in different age groups. ${ }^{9}$ In addition, younger individuals have a higher rate of cell proliferation in thyroid tissue, which may partly explain the greater sensitivity of this group to radioiodine, ${ }^{10}$ even in cases with distant metastasis. Similarly, it is possible that specific genetic mutations are present in this group of patients, providing better survival, although there is no description of a well-recognized genetic pattern yet. ${ }^{11}$

\section{Conclusion}

We observed a distinct pattern of clinicopathological manifestation of thyroid cancer according to age groups. Individuals in extremes of age resemble in several pathological 
Table 2 Pattern of histopathological presentation of well-differentiated thyroid carcinoma according to age

\begin{tabular}{|c|c|c|c|c|c|c|c|c|c|c|c|c|}
\hline & & \multicolumn{10}{|c|}{ Age } & \multirow[t]{2}{*}{$p$ value } \\
\hline & & \multicolumn{2}{|c|}{$\leq 24$ yo } & \multicolumn{2}{|c|}{$25-44$ yo } & \multicolumn{2}{|c|}{ 45-64 yo } & \multicolumn{2}{|c|}{$\geq 65$ yo } & \multicolumn{2}{|c|}{ Total } & \\
\hline \multicolumn{2}{|l|}{ Mean (SD) } & \multicolumn{2}{|c|}{$20.10(2.79)$} & \multicolumn{2}{|c|}{$35.78(5.75)$} & \multicolumn{2}{|c|}{$53.25(5.20)$} & \multicolumn{2}{|c|}{71.05 (5.39) } & \multicolumn{2}{|c|}{$47.75(14.32)$} & - \\
\hline \multicolumn{2}{|c|}{$\begin{array}{l}\text { Tumoral Diameter } \\
\text { Median (Min-Max) }\end{array}$} & \multicolumn{2}{|c|}{$1.9(0.1-7)$} & \multicolumn{2}{|c|}{$1.5(0.1-7)$} & \multicolumn{2}{|c|}{$1.2(0.04-7.6)$} & \multicolumn{2}{|c|}{$1.25(0.1-10)$} & \multicolumn{2}{|c|}{$1.3(0.04-10)$} & $<0.001$ \\
\hline & & $\mathrm{N}$ & $\%$ & $\mathrm{~N}$ & $\%$ & $\mathrm{~N}$ & $\%$ & $\mathrm{~N}$ & $\%$ & $\mathrm{~N}$ & $\%$ & - \\
\hline \multirow[t]{2}{*}{ Gender } & Male & 5 & 17.2 & 35 & 17.6 & 46 & 17.7 & 18 & 24.0 & 104 & 18.5 & \multirow[t]{2}{*}{0.633} \\
\hline & Female & 24 & 82.7 & 163 & 82.3 & 213 & 82.2 & 57 & 76.0 & 457 & 81.4 & \\
\hline \multirow[t]{2}{*}{ Histology (CA) } & Papillary & 26 & 89.6 & 192 & 96.9 & 248 & 95.7 & 64 & 85.3 & 530 & 94.4 & \multirow[t]{2}{*}{$<0.001$} \\
\hline & Follicular & 3 & 10.3 & 6 & 3.0 & 11 & 4.2 & 11 & 14.6 & 31 & 5.5 & \\
\hline \multicolumn{2}{|c|}{$\begin{array}{l}\text { Neural or lymphovascular } \\
\text { invasion }\end{array}$} & 8 & 27.5 & 22 & 11.1 & 21 & 8.1 & 12 & 16.0 & 63 & 11.2 & 0.007 \\
\hline \multicolumn{2}{|l|}{ Multifocality } & 9 & 31.0 & 75 & 37.8 & 99 & 38.2 & 23 & 30.6 & 206 & 36.7 & 0.574 \\
\hline \multicolumn{2}{|c|}{ Gland capsule invasion } & 22 & 75.8 & 103 & 52.0 & 134 & 51.7 & 39 & 52.0 & 298 & 53.1 & 0.097 \\
\hline \multicolumn{2}{|c|}{ Extraglandular invasion } & 12 & 41.3 & 50 & 25.2 & 73 & 28.1 & 28 & 37.3 & 163 & 29.0 & 0.111 \\
\hline \multicolumn{2}{|l|}{ Positive neck LN } & 12 & 41.3 & 51 & 25.7 & 55 & 21.2 & 9 & 12.0 & 127 & 22.6 & 0.008 \\
\hline \multicolumn{2}{|c|}{$\begin{array}{l}\text { Lateral compartment positive } \\
\text { LN }\end{array}$} & 4 & 29.0 & 24 & 12.1 & 15 & 5.7 & 6 & 8.0 & 49 & 8.7 & 0.081 \\
\hline
\end{tabular}

Abbreviations: CA, Carcinoma; LN, Lymph node; PD, Poorly differentiated; yo, years old.

Note: Variables were expressed in absolute $(\mathrm{N})$ and relative frequencies (\%); Mean (Standard Deviation) or Median (Minimum to maximum) were used according to appropriate; diameter was expressed in centimeters; $p=$ level of significance. Post-hoc analysis followed Kruskal-Wallis test when analyzing diameter. Statistically significant differences were observed between: $25-44$ yo and $45-64$ yo $(p=0.002) ; 45-64$ yo and $\geq 65$ yo $(p=0.002)$; $\leq 24$ yo and 45-64 yo $(p=0.015)$.

features, although literature data have shown survival is not related to the clinicopathological pattern at early ages. In our sample, young people with thyroid cancer presented with features of more aggressive disease.

\section{References}

1 Edge SE, Byrd DR, Carducci MA, Compton CA. AJCC Cancer Staging Manual. 7th ed. New York: Springer; 2010

2 Hundahl SA, Fleming ID, Fremgen AM, Menck HR. A National Cancer Data Base report on 53,856 cases of thyroid carcinoma treated in the U.S., 1985-1995. Cancer 1998; 83(12):2638-2648

3 Gilliland FD, Hunt WC, Morris DM, Key CR. Prognostic factors for thyroid carcinoma. A population-based study of 15,698 cases from the Surveillance, Epidemiology and End Results (SEER) program 1973-1991. Cancer 1997;79(3):564-573

4 Miccoli P, Minuto MN, Ugolini C, et al. Papillary thyroid cancer: pathological parameters as prognostic factors in different classes of age. Otolaryngol Head Neck Surg 2008;138(2):200-203
5 Ito Y, Miyauchi A, Kihara M, Higashiyama T, Kobayashi K, Miya A. Prognostic significance of young age in papillary thyroid carcinoma: analysis of 5,733 patients with 150 months' median followup. Endocr J 2014;61(5):491-497

6 Ito Y, Miyauchi A, Tomoda C, Hirokawa M, Kobayashi K, Miya A. Prognostic significance of patient age in minimally and widely invasive follicular thyroid carcinoma: investigation of three age groups. Endocr J 2014;61(3):265-271

7 Sampson E, Brierley JD, Le LW, Rotstein L, Tsang RW. Clinical management and outcome of papillary and follicular (differentiated) thyroid cancer presenting with distant metastasis at diagnosis. Cancer 2007;110(7):1451-1456

8 Shirahige Y, Ito M, Ashizawa K, et al. Childhood thyroid cancer: comparison of Japan and Belarus. Endocr J 1998;45(2):203-209

9 Haymart MR. Understanding the relationship between age and thyroid cancer. Oncologist 2009;14(3):216-221

10 Saad AG, Kumar S, Ron E, et al. Proliferative activity of human thyroid cells in various age groups and its correlation with the risk of thyroid cancer after radiation exposure. J Clin Endocrinol Metab 2006;91(7):2672-2677

11 Sassolas G, Hafdi-Nejjari Z, Ferraro A, et al. Oncogenic alterations in papillary thyroid cancers of young patients. Thyroid 2012; 22(1):17-26 